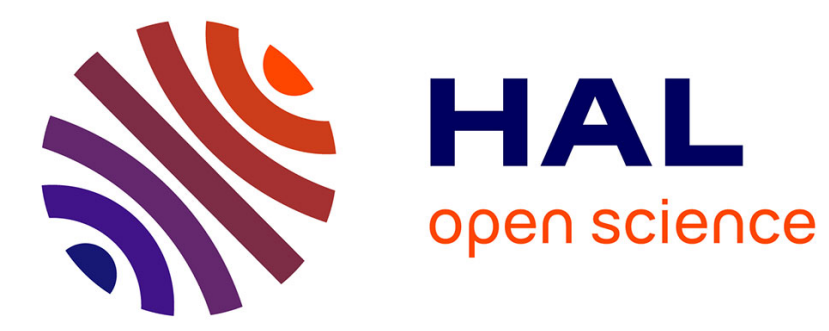

\title{
Collegialité et bureaucratie dans les firmes américaines d'avocats d'affaires
}

Emmanuel Lazega

\section{To cite this version:}

Emmanuel Lazega. Collegialité et bureaucratie dans les firmes américaines d'avocats d'affaires. Droit et Société, 1993, 23/24, pp.15-39. 10.3406/dreso.1993.1202 . hal-01009791

HAL Id: hal-01009791

https://hal-sciencespo.archives-ouvertes.fr/hal-01009791

Submitted on 18 Jun 2014

HAL is a multi-disciplinary open access archive for the deposit and dissemination of scientific research documents, whether they are published or not. The documents may come from teaching and research institutions in France or abroad, or from public or private research centers.
L'archive ouverte pluridisciplinaire HAL, est destinée au dépôt et à la diffusion de documents scientifiques de niveau recherche, publiés ou non, émanant des établissements d'enseignement et de recherche français ou étrangers, des laboratoires publics ou privés. 


\title{
Collégialité et bureaucratie dans les firmes américaines d'avocats d'affaires *
}

\author{
Emmanuel Lazega ***
}

\section{Summary}

\section{Collegiality and Bureaucracy in American Corporate Law Firms}

This article summarizes existing literature pertaining to the organizational form of American corporate law firms (the Cravath model), and the current changes experienced by such firms resulting from market pressures and internal growth processes. These changes correspond to the bureaucratization of structure (increased hierarchical organization, departmentalization, polycentric arrangements) and management policies (concerning such issues as work flow, compensation, marketing, evaluation of work quality, management of associates). The author also describes the resistance offered by the collegial form to this bureaucratization, as well as the emergence of new organizational forms (mega-firms, franchized firms, multidisciplinary partnerships), and the resulting debates concerning the redefinition of professionalism. Given the future uncertainty of the American model in the United States context and the spreading of the Cravath model in continental Europe, European firms will undoubtedly encounter similar changes and difficulties.

\section{Résumé}

Cet article résume la littérature sur la forme organisationnelle des firmes américaines d'avocats d'affaires (le modèle Cravath) et sur les changements que connaissent actuellement ces firmes sous la pression du marché et des processus internes de croissance. Ces changements correspondent à une bureaucratisation à la fois en termes de structure (hiérarchisation, départementalisation, arrangements polycentriques) et de politiques de gestion (distribution des dossiers, partage des bénéfices entre associés, marketing, évaluation de la qualité du travail, gestion des stagiaires). L'auteur décrit aussi la résistance que la forme collégiale classique oppose à cette bureaucratisation, ainsi que l'émergence de formes organisationnelles nouvelles (méga-firmes, franchiseurs, associations multidisciplinaires) et les débats qui s'ensuivent concernant une redéfinition du professionnalisme. Dans la mesure où le modèle américain, dont l'avenir même est incertain aux États-Unis, se diffuse aujourd'hui en Europe continentale, les firmes européennes pourraient aller au devant de changements et de difficultés semblables.

\section{L'auteur}

Maître de conférences à l'Université de Versailles. Ses recherches actuelles portent sur les réseaux intra- et interorganisationnels, ainsi que sur la contribution de l'approche structurale à la sociologie des organisations et à la sociologie économique. Parmi ses publications récentes, on peut citer : The Micropolitics of Knowledge, New York, Aldine-de Gruyter, 1992; "Analyse de réseau d'une organisation collégiale : les avocats d'affaires ", Revue française de sociologie, 1992, 33 : 559589; «Acteurs, cibles et leviers : analyse factorielle des relations de contrôle indirect dans une firme américaine d'avocats d'affaires ", Bulletin de méthodologie sociologique, 1992, 37 : 41-51, avec Stéphane Vari.

* Cette étude est extraite de la monographie d'une firme d'avocats de Nouvelle-Angleterre et, plus généralement, d'un travail consacré à la structure et au fonctionnement des organisations collégiales, ainsi qu'au pouvoir entre égaux. Je remercie Benoit Bastard des nombreuses suggestions apportées à ce texte. ** Laboratoire d'Analyse Secondaire et de Méthodes Appliquées en Sociologie (LASMAS). 


\section{E. Lazega}

Collégialité et bureaucratie dans les firmes américaines d'avocats d'affaires

1. Le terme « association » traduit ici la notion anglo-saxonne de partnership. Il s'agit d'une société de personnes ou d'une société en nom collectif. Dans cette forme légale spécifique, les associés (ou partners) sont copropriétaires de leur firme et entièrement co-responsables de ses actions. Pour éviter une possible confusion, il faut rappeler que le terme anglais partner se traduit par le terme français « associé » et que le terme associate se traduit par "stagiaire».

2. En tant que forme juridique et économique, le partnership contient un principe de collégialité et d'obligation mutuelle. Théoriquement, il présuppose donc des relations étroites entre membres, et les firmes sont parfois comparées par les associés à des familles (a family away from the family). Même dans les firmes dirigées par des systèmes de gouvernance très fermés ou très peu " démocratiques ", malgré la croissance en taille, la différenciation interne et la stratification de l'association, les leaders doivent toujours faire un effort pour préserver le consensus dans une organisation très volatile. Cependant, en tant que notion sociologique, le terme « collégialité » renvoie autant à une forme organisationnelle qu'à un type de relation ou un code de conduite entre associés.
Les firmes américaines d'avocats d'affaires construites sur le modèle Cravath représentent une forme organisationnelle vieille d'un siècle et une synthèse originale de collégialité et de bureaucratie. Ces associations ${ }^{1}$ de grande taille rassemblent, entre autres, et suivant une expression de Bourricaud (1961), des « associés-rivaux condamnés à vivre ensemble ». Elles sont efficaces dans la production de services juridiques aux entreprises, engendrant parfois d'énormes profits. Cet article utilise une approche organisationnelle pour décrire cette forme et les changements qu'elle subit depuis une trentaine d'années. Il se base sur la littérature anglo-saxonne disponible à ce sujet - en particulier sur les travaux de Galanter et Palay (1991), Gilson et Mnookin $(1985,1989)$ et Nelson (1988) - et sur une recherche conduite dans six firmes de Nouvelle Angleterre entre 1988 et 1991.

La forme organisationnelle de ces cabinets américains d'avocats d'affaires s'est beaucoup diversifiée depuis le début du siècle. On s'intéressera ici essentiellement aux firmes de taille moyenne (pour les États-Unis), c'est-à-dire pouvant rassembler en général plusieurs centaines d'avocats. Le contraste entre ces firmes et, par exemple, les mégafirmes intercontinentales actuelles reste très important. Ces dernières étant encore peu étudiées d'un point de vue organisationnel, on ne cherchera pas ici à généraliser les connaissances dont on dispose sur les changements apportés aux firmes de taille moyenne au cours des trente dernières années, essentiellement leur croissance et leur bureaucratisation.

Cette forme organisationnelle cristallise un équilibre entre les forces parfois contradictoires du marché, de la profession et de l'État qui pèsent sur la pratique et le commerce du droit. En ce sens, une telle approche espère contribuer aux réflexions actuelles sur la redéfinition du «professionnalisme » et des mécanismes d'auto-régulation dans les professions juridiques. Parce que cette forme se diffuse aujourd'hui en Europe, les questions qu'elle soulève de ce point de vue sont aussi d'actualité pour la profession juridique française.

\section{Entre collégialité et bureaucratie}

Le modèle Cravath organise la production de services juridiques aux entreprises d'une manière qui adapte aux avocats d'affaires les contraintes de la profession. Ce modèle relève de la forme collégiale, au sens wébérien revu par Waters (1989) ${ }^{2}$. Ce dernier définit la forme collégiale de la manière suivante: une structure collégiale idéaltypique est une organisation à l'intérieur de laquelle domine une orientation vers le consensus entre membres d'une profession théoriquement égaux dans leur niveau d'expertise, mais spécialisés dans des domaines différents. Les caractéristiques organisationnelles qu'implique cette définition sont les suivantes : 1) ces organisations utilisent et appliquent une connaissance théorique; 2) leurs membres sont considérés comme des professionnels et leur carrière est diffé- 
renciée en deux étapes au moins, d'apprentissage et de pratique; 3) bien qu'orientées vers la performance, elles rencontrent des difficultés lorsqu'elles doivent comparer la performance des spécialistes, d'où l'instauration d'une égalité formelle entre eux; 4) elles se contrôlent elles-mêmes; 5) elles se donnent des méthodes de contrôle de la production (échanges d'avis entre collègues, par exemple); 6) elles ont des forums collectifs - un système de commissions plus ou moins complexe et hiérarchisé - où les décisions sont prises. Ces propriétés sont idéal-typiques, tout comme celles de l'organisation bureaucratique classique. De fait, collégialité et bureaucratie coexistent dans toutes les structures de prise de décision.

Ces caractéristiques idéal-typiques différencient clairement l'organisation collégiale de l'organisation bureaucratique. Cependant, des variations empiriques produisent en fait trois types d'organisation collégiale: a) les organisations exclusivement collégiales (les tâches administratives sont assumées par les collègues); b) les organisations à prédominance collégiale (l'activité professionnelle est centrale et l'activité administrative lui est subordonnée; c'est l'organisation autonome professionnelle de Scott (1965)); c) les organisations collégiales intermédiaires (où les employés professionnels sont clairement subordonnés à la structure administrative; c'est l'organisation hétéronome professionnelle de Scott). Il s'ensuit que, dans la plupart des cas, la collégialité n'est pas le seul principe qui structure les modalités de prises de décision ou d'action collective, mais qu'elle coexiste avec la bureaucratie.

La structure formelle des partnerships américains d'avocats d'affaires relève le plus souvent du deuxième type, bien que la synthèse spécifique que représente chaque firme puisse se rapprocher plus ou moins du troisième. Ainsi, avec la croissance de la firme, la responsabilité de fonctions particulières est attribuée à des associés spécifiques. Un système de commissions émerge, dont la lourdeur peut être compensée, par exemple, par une structure de gestion plus pyramidale où les décisions au jour le jour sont prises par un directeur ( $m a$ naging partner). Le pouvoir de ce dernier est souvent considérable, bien que maintenu sous contrôle par les décisions que se réserve le partnership au complet, et par l'influence des associés qui représentent des clients importants.

Concrètement, la forme organisationnelle des firmes d'avocats américaines de taille moyenne, ainsi que sa genèse, sont décrites en détail par Smigel (1969), Nelson (1988), Galanter et Palay (1991), et bien d'autres ${ }^{3}$. Comme l'indique plus haut la définition de Waters, la structure formelle de ces firmes est basée premièrement sur la distinction entre administratifs et professionnels, et deuxièmement sur la distinction, parmi les professionnels, entre associés (copropriétaires de la firme) et stagiaires (avocats salariés tenus de facturer un certain nombre d'heures de travail par an). Les associés trônent au sommet de la hiérarchie et constituent une sorte d'oligarchie qui se reproduit
Droit et Société 23/24-1993

3. Pour la place de ces firmes dans le contexte de la profession juridique américaine, voir par exemple Laumann et Heinz (1977), Heinz et Laumann (1982), Kagan et Rosen (1985), Halliday (1987), Abel et Lewis (1988, 1989), Osiel (1990), Sterett (1990). 


\section{E. Lazega}

Collégialité et bureaucratie dans les firmes américaines d'avocats d'affaires par cooptation. Ils dirigent la firme au moyen d'un système complexe de commissions. La relation hiérarchique entre associés et stagiaires est d'ordinaire très marquée. Les stagiaires doivent manifester une certaine déférence à l'égard des associés, surtout en public ou devant les clients. Cette structure collégiale formelle est lourde, difficile à mobiliser, et les prises de décisions sont lentes. Face à ces difficultés, les firmes développent des structures informelles plus centralisées, dirigées par des associés plus centraux que d'autres (par exemple les rainmakers, qui «contrôlent» les plus gros clients de la firme), ou une centralisation plus bureaucratique.

Les forces du marché et les pressions internes ont provoqué une forte croissance au cours des trente dernières années. Nelson (1988) distingue deux formes traditionnelles de croissance : par représentation générale ou spécialisée. En général, les firmes cherchent à suivre l'une et l'autre voie, essayant de s'étendre dans un champ spécialisé puis de consolider en attirant le reste du travail juridique du client, ou de capitaliser sur des spécialités développées pour certains clients en les offrant à d'autres. Le passage d'une voie à l'autre pose des problèmes éthiques de conflits d'intérêt (Hazard et Rhode, 1988; Lazega, 1993) sur lesquels nous revenons plus bas. La combinaison de spécialités développée par un cabinet spécifique est le produit de liens tissés par le passé avec des clients, des normes concernant ce qui constitue un travail acceptable, des règles formelles concernant la représentation multiple, et des opportunités offertes par l'émergence de nouveaux domaines d'expertise.

Les deux grands domaines de spécialisation de ces firmes sont les contentieux (litigation) et le conseil d'entreprise (ou corporate, i.e. tous les services juridiques, excepté les contentieux, dont ont besoin les entreprises et les institutions). Dans l'un et l'autre domaines, les tâches accomplies par les avocats peuvent créer entre eux une forte interdépendance.

Le travail des avocats conseils d'entreprises (corporate lawyers) comporte un large éventail de tâches aussi vaste que mettre sur pied des transactions complexes, comme par exemple l'achat d'un centre commercial, arranger des accords de prêts bancaires, créer des sociétés, contrôler l'achat ou la vente de produits financiers, diriger pour un client des négociations à l'échelle internationale, et bien d'autres encore. Toutes ces tâches peuvent essentiellement se ramener à deux : l'écriture de documents (souvent le travail des stagiaires) et la négociation de leur forme finale (souvent le travail des associés, qui consiste à transformer un accord conçu de manière unilatérale en un accord consensuel avec l'autre partie). Les conseils que le client demande ou reçoit des avocats dépassent souvent le domaine des compétences juridiques de ces derniers, comme dans le cas de programmes d'investissements. Il est important de voir que la mémoire collective de la firme est une ressource indispensable que les stagiaires et 
les associés souhaitent pouvoir mobiliser facilement au cours du processus d'écriture et de négociation de ces documents.

Les litigators sont des spécialistes du contentieux plus combatifs, qui interviennent après coup pour représenter le client dans un conflit ouvert avec l'autre partie. Leur activité est moins feutrée et continue que celle de la plupart des avocats conseils. Elle est plus irrégulière, très intense pendant une période fixée par les délais de procédure, puis parfois beaucoup moins soutenue pendant de longues périodes. Elle consiste à représenter le client auprès du tribunal pendant le procès, y compris assigner l'autre partie en justice, gérer les pièces introduites dans les procès, écrire et présenter des requêtes. Leur travail peut comprendre des tâches comme la reformulation en termes juridiques des prétentions de leurs clients, donner à celles-ci une tournure juridique, argumentée de manière serrée, ne contenant pas d'information jugée non pertinente. Mais le moment le plus libre de leur activité se situe avant le début du procès. C'est la période où les jeux de pouvoir et la gestion de l'information préorganisent le procès en négociant la définition du conflit (Mann, 1985). Dans les grandes firmes qui gèrent des contentieux à grande échelle, l'organisation du travail des avocats suppose la création d'équipes plus ou moins temporaires d'associés et de stagiaires, ainsi qu'une infrastructure qui peut devenir complexe, comme par exemple la création d'équipes satellites qui travaillent à plein temps dans les locaux du client.

La nature du travail juridique a aussi changé au cours de cette période. En particulier, les avocats employés par les entreprises ellesmêmes (in-house lawyers) prennent en charge le travail juridique le plus routinier de leur entreprise. Ils sous-traitent le travail complexe exigeant davantage de sophistication, de spécialisation ou d'expérience, à un ou plusieurs grands cabinets. Une partie considérable du travail de ces derniers relève donc du contentieux, discipline qui exige des investissements imprévisibles et intensifs en temps, ou de domaines du droit très techniques, complexes ou en constant changement. La tendance est aujourd'hui à la spécialisation des grands cabinets dans les domaines qui exigent des efforts imprévisibles 4 .

Ces firmes justifient des honoraires très élevés en se présentant comme l'élite de la profession juridique, seule capable de mobiliser rapidement de grandes équipes d'avocats et de gérer des affaires complexes exigeant une connaissance sophistiquée du droit et de ses plus récents développements. Pour recruter les meilleurs stagiaires possibles (i.e. sortant des facultés de droit les plus réputées), ces firmes doivent pouvoir leur donner l'espoir de devenir associés au terme de six à dix ans de travail comme stagiaires. Si au terme de cette période le stagiaire n'est pas coopté comme associé, il ou elle doit quitter la firme. C'est la règle du up or out. Pour Gilson et Mnookin (1985), les études d'avocats s'organisent autour de la promotion au rang d'associé comme mécanisme de protection de leur capital humain et social, ainsi que de contrôle des stagiaires. Pour ces auteurs, la res-
4. Ceci a des effets sur la distribution du travail et les flux de dossiers au sein de la firme. Ces flux passent principalement par deux étapes: l'ouverture de dossiers et la délégation des tâches qui s'ensuit. Ces deux étapes sont prises en charge par des procédures qui sont plus ou moins formelles et flexibles, plus ou moins respectées par les avocats eux-mêmes, et variables parfois d'un département à l'autre. Cette flexibilité est impérative précisément parce que la distribution du travail découle de la nature très variable des tâches, de la provenance du dossier, des exigences du client, du personnel disponible. Le contrôle des incertitudes engendrées par cette flexibilité est une source de pouvoir supplémentaire dans ce type d'organisation. 


\section{E. Lazega}

Collégialité et bureaucratie dans les firmes américaines d'avocats d'affaires source la plus importante d'une étude d'avocats réside dans son capital humain et social (expérience, niveau d'expertise et de sophistication, réputation, bonnes relations avec les clients, bonnes relations entre associés). La structure traditionnelle des firmes d'avocats a pour fonction de rendre possible le contrôle, le partage et l'accumulation de ce capital.

$\mathrm{Au}$ cours des trente dernières années, cette règle - ajoutée à un contexte économique et politique favorable - a poussé les firmes à une croissance qui a assuré leur intégration à court terme (puisqu'il était facile de recruter des stagiaires et de leur promettre l'accession rapide au rang d'associé), mais qui l'a aussi menacée à long terme. Aujourd'hui, en période de crise, l'un des principaux problèmes de ces firmes est de trouver un nouveau moyen de contrôler leur croissance et de protéger leur expertise. L'intégration au moyen de la promotion au rang d'associé est menacée par les limites du marché, notamment par la taille de ces firmes et par la concurrence entre elles. En réaction à ce danger, elles se bureaucratisent davantage. Ces organisations collégiales ont récemment traversé, ou traversent encore, une période de restructuration. Les règlements internes s'épaississent, le nombre des commissions baisse, les firmes se départementalisent et partagent entre un nombre de plus en plus restreint d'avocats des responsabilités administratives et commerciales importantes. Les critères du partage des bénéfices tendent à se diversifier : les firmes où ce partage était basé encore récemment sur l'ancienneté des associés donnent pour la plupart davantage de poids à la performance, avec des manières très pragmatiques de la mesurer (nombre d'heures facturées, nombre de clients ramenés, nombre d'heures consacrées aux responsabilités administratives). Cette bureaucratisation consiste à standardiser et à routiniser un maximum de tâches (par exemple examiner des caisses entières de documents pour déterminer s'il faut laisser l'autre partie y accéder, résumer des dépositions, faire enregistrer des documents, etc.) et à les assigner à des employés " para légaux ». Elle accroît la partie administrative de la firme et le nombre de ces employés. Elle transfert aussi davantage d'autorité à des gestionnaires qui ne sont pas avocats. Signe supplémentaire de la bureaucratisation et du changement dans la nature des relations entre membres, de nombreuses firmes envisagent aussi d'implanter certaines formes d'évaluation formelle de la qualité de la production des associés, en plus des contrôles comptables. Dans certaines firmes, des associés doivent changer de spécialité et en choisir de plus lucratives.

L'ensemble des associés conserve le plus souvent le contrôle des décisions les plus importantes, mais la plupart des firmes envisagent la création de différents types d'associés et de positions de salariés permanents. Toutes recrutent de plus en plus de manière latérale. Cette politique est considérée comme rentable (la firme investit moins dans la formation des stagiaires), mais elle modifie aussi le fonctionnement relationnel des membres. La concurrence entre associés et en- 
tre stagiaires se fait plus dure, et la coopération prend des formes nouvelles. Certaines firmes présentent la création de ces différents statuts et le recrutement latéral comme des moyens de contrôle de leur croissance. Mais dans la mesure où leurs affaires dépendent d'une économie relativement imprévisible, et dans la mesure où il serait dangereux pour ces sociétés d'enrayer le mécanisme de l'accession au rang d'associé, l'idée même qu'elles contrôlent réellement leur croissance semble douteuse.

Nelson (1988) a décrit de manière approfondie cette période de transition que connaissent aujourd'hui les firmes américaines, ainsi que certaines formes d'équilibre entre collégialité et bureaucratie qu'elles cherchent à établir. Ces changements, présentés souvent comme réduisant le coût des prises de décision collectives, rencontrent des résistances, bien qu'ils soient perçus comme une condition de survie. En effet, le processus de bureaucratisation a des limites. Les idéaux du professionnalisme qui servent de culture de motivation, la complexité et le caractère non routinier des tâches, ainsi que la relative imprévisibilité du comportement des clients sont trois facteurs qui expliquent, selon Nelson (1988), que les grandes firmes, dites general practice parce que leur expertise est censée couvrir la plupart des domaines du droit, ne soient pas destinées à se bureaucratiser encore davantage. Reste que ces changements n'en constituent pas moins une menace pour la manière dont la profession juridique conduit ses affaires. Avec la bureaucratisation et la remise en question de la règle du up or out, cette forme de gestion du capital humain et social que constitue le partnership est aussi menacée. La crainte du comportement perçu comme opportuniste (mobilité des associés, absence de partage des clients, des compétences) augmente à mesure que des associés quittent la firme, emmenant souvent avec eux des clients importants. La question de l'intégration est donc un problème connu des membres et géré d'une manière qui en menace la forme organisationnelle actuelle. Même et surtout bureaucratisées, ces structures formelles ne sont pas capables à elles seules d'assurer l'intégration de la firme (Lazega, 1992b). Du fait de changements à la fois internes et externes, les grandes études d'avocats se cherchent donc de nouvelles formes organisationnelles, plus adaptées à leur environnement, et permettant de contrôler davantage leur croissance. Les pages suivantes décrivent plus systématiquement les paramètres organisationnels sur lesquels les firmes jouent pour maintenir un équilibre entre bureaucratie et collégialité.

\section{Le processus de bureaucratisation}

Cette partie examine plus en détail la bureaucratisation croissante de ces firmes, de même que les limites de ce processus. La croissance, telle qu'elle a été expérimentée par les partnerships américains pendant les vingt dernières années, provoque des changements dans la 


\section{E. Lazega}

Collégialité et bureaucratie dans les firmes américaines d'avocats d'affaires manière dont ces firmes fonctionnent. On décrit d'abord leur structure formelle (le système de commissions et la hiérarchie) avant d'évoquer la transformation de leur gestion.

\section{a) Hiérarchisation, départementalisation et poly- centrisme}

$\mathrm{Au}$ début des années 1990, les firmes américaines de taille moyenne n'ont pas toutes atteint un degré très élevé de bureaucratisation. Cependant, quels que soient les différents scénarios de réorganisation décrits par la littérature, ce changement est caractérisé au moins par trois processus : l'établissement d'une nouvelle hiérarchie formelle, une départementalisation et une relative décentralisation.

Le degré de hiérarchisation permet de distinguer les firmes qui conservent l'informalité des relations et celles qui définissent des chaînes de responsabilités claires avec, par exemple, des directeurs de département très influents, des procédures et des contrôles rigides. Mais au fur et à mesure de leur croissance et de leur spécialisation, ces organisations développent des structures internes de décision, de coordination et de contrôle (governance structures) de moins en moins participatives qui se greffent sur le système des commissions. Certains groupes, par exemple, se détachent et assument la fonction de planification stratégique visant à protéger la clientèle déjà acquise et à profiter de nouvelles opportunités. Dans presque toutes les firmes, l'assemblée des associés conserve le contrôle ultime des décisions les plus importantes (comme la nomination d'un nouvel associé). Presque toutes ont adopté un managing partner, ou un équivalent fonctionnel, qui consacre une plus ou moins grande partie de son temps à des tâches de gestion et qui ne dispose pratiquement jamais de pouvoirs tyranniques. Il se présente plutôt comme un homme de consensus, un coordinateur ou un planificateur.

Le nombre de commissions exerçant des fonctions essentielles (exécutif, finance, recrutement, gestion des stagiaires, personnel nonprofessionnel, etc.) a tendance à baisser. Celles qui se situent au sommet de la pyramide de gestion et où se concentre le pouvoir (comme la commission exécutive, ou celle des finances dont la principale fonction est la distribution annuelle des bénéfices) se composent souvent des mêmes associés. La force de la partie administrative varie aussi de firme en firme. Certaines se donnent des administrateurs faibles et spécialisés, d'autres des entrepreneurs forts qui rendent directement des comptes au managing partner ou à la commission exécutive. Il est rare cependant, même lorsqu'ils sont eux-mêmes des associés (souvent sans clientèle), qu'ils traitent d'égal à égal avec le managing partner ou le président de la commission des finances.

Cependant une tension permanente existe entre l'autorité du gestionnaire et la responsabilité vis-à-vis du client. Comme le montre en particulier Nelson (1988), les positions d'autorité formelle sont fragi- 
les dans ces cabinets comme dans d'autres organisations collégiales. Le pouvoir peut dépendre davantage de la capacité de l'associé à contrôler des clients importants que de la capacité de créer un consensus entre associés sur la façon de gérer la firme. Le contrôle des gros clients permet à un associé d'exercer sur ses collègues des pressions en cascade, toutes présentées comme découlant des exigences du marché. La stratification informelle de la firme (on y revient plus bas) interfère de manière incontournable avec sa hiérarchie formelle. D'autres sources d'autorité (réputation, ancienneté) comptent dans une firme et sont utilisées dans un contexte où la manipulation des relations informelles par les associés témoigne d'une «culture stratégique » très sophistiquée (Lazega, 1992b; Lazega et Vari, 1992).

La départementalisation d'une firme est souvent présentée comme un pas important dans sa réorganisation. Elle introduit des règles, des procédures et des changements pratiques dans la manière de travailler qui dessaisissent les associés d'un certain contrôle sur leurs pratiques quotidiennes et transforment la structure des carrières des stagiaires. La création de départements (par exemple litigation ou corporate) et la délégation de fonctions importantes à leur directeur clarifient les responsabilités vis-à-vis des clients ou des associés. Les directeurs de départements, même s'ils ne comptent pas toujours parmi les associés les plus visiblement rentables de la firme, jouent un rôle important du point de vue du recrutement, de la formation et de la promotion des stagiaires, du point vue du contrôle et de la gestion du capital humain, ainsi que des activités de marketing. Il faut noter que certains domaines du droit américain (corporate law) sont difficiles à départementaliser en tant que tels parce qu'ils rassemblent différentes disciplines (general corporate law, banking, securities, labor, etc.) et exigent la mise en place de systèmes flexibles de collaboration et de coordination. Cette départementalisation permet aussi aux gestionnaires de la firme, bien qu'ils s'en défendent souvent officiellement, de considérer chaque département comme un centre de profit. Ces comparaisons entre profits engendrés par les uns et les autres sont souvent basés sur des observations à long terme pour éviter les tensions interdépartementales. La rigidité et les frontières internes introduites par ce processus n'améliorent pas toujours la capacité des firmes de s'adapter aux besoins conjoncturels de marchés qui exigent des services interdisciplinaires.

L'ouverture de plus en plus fréquente de bureaux dans d'autres villes ou pays, ou la fusion entre firmes différentes, créent des structures plus ou moins polycentriques. Les firmes doivent choisir entre considérer ou non chaque bureau comme un centre de profit séparé. Elles doivent établir un équilibre entre la centralisation administrative et la décentralisation de fait due à la spécificité de chaque marché. Certaines firmes sont plus centralisées que d'autres, en particulier lorsque l'un des bureaux est plus gros que les autres. Les difficultés liées à cette configuration polycentrique sont nombreuses. D'un bu- 


\section{E. Lazega}

Collégialité et bureaucratie dans les firmes américaines d'avocats d'affaires reau à l'autre, les salaires et méthodes de recrutement varient. Les bureaux tendent à développer ou à conserver des normes idiosyncratiques, surtout après une fusion; les membres s'utilisent mutuellement non pas sur la base de leurs compétences, mais sur celle des liens développés sur place avec des associés locaux; les flux d'informations, de conseils et de clients entre bureaux sont rarement symétriques; l'évaluation des stagiaires se fait de manière plus locale; la supervision des flux de dossiers par département à des fins de contrôle éthique et de gestion est plus difficile. L'allégeance des associés à leurs clients peut dépasser leur loyauté à l'égard de leur propre firme. Il n'est pas possible de voir, dans la littérature actuelle, dans quelle mesure les politiques de gestion peuvent polariser les firmes et transformer des bureaux aux intérêts différents en blocs opposés. Ceci pose la question de la cohésion interne de ces firmes, sur laquelle nous revenons plus bas.

\section{b) Nouvelles politiques de gestion}

Dans la mesure où elles remettent en question le statut professionnel des avocats qui travaillent dans ces grandes firmes, les politiques de gestion ont fait l'objet d'une attention particulière. On prendra ici l'exemple de cinq domaines dans lesquels des changements de politique accompagnent les changements de structure: la planification stratégique, le partage des profits, le marketing, l'évaluation par les pairs et la gestion des stagiaires.

Quelle que soit leur taille, les firmes risquent de perdre le contrôle de leur croissance. La planification stratégique représente un effort de systématisation de la gestion des cette croissance. La manière traditionnelle de planifier et d'assurer un profit élevé était de se maintenir à une taille un peu trop petite et d'avoir toujours un peu trop de travail. Au cours des années 1970-1980 (période de forte croissance de la demande), c'est le rapport entre le nombre de stagiaires et le nombre d'associés (leverage) qui servait d'indicateur de contrôle. Aujourd'hui le recrutement se base sur les projections des associés sur un à trois ans. Les firmes cherchent donc à contrôler ce processus en essayant de prédire les besoins futurs et en planifiant, parfois de manière très détaillée, la manière dont elles veulent croître. Plusieurs facteurs, dont l'incertitude économique et le turnover des stagiaires, rendent cependant cette planification difficile. La sophistication de cette planification stratégique varie de firme en firme, notamment avec le degré de complexité de l'infrastructure et le rôle joué par les directeurs de départements et leur connaissance approfondie du marché de leur spécialité.

En période de crise, les firmes sont tentées de résister à la pression de la croissance. La planification consiste alors à s'en tenir à une taille juste suffisante pour prendre en charge le travail juridique des gros clients institutionnels. Le problème devient dès lors celui du re- 
crutement des stagiaires qui voient dans ce contexte leurs chances de devenir associés diminuer encore davantage, et les critères de leur sélection dépendre encore plus de l'économie plutôt que de la qualité de leur travail. A moyen terme, c'est le capital humain de la firme qui s'en trouve menacé. C'est pourquoi elles ont recours aujourd'hui à des mesures pratiques qui cherchent à éviter une remise en question systématique de la règle du up or out. Elles deviennent encore plus sélectives en matière d'ouverture des dossiers. Elles recrutent «latéralement » plutôt qu'à la sortie des facultés de droit. Elles exigent davantage de «flexibilité » de la part de leurs avocats qui sont parfois " encouragés » à changer de spécialité pour s'adapter aux demandes de marchés plus lucratifs. Elles recrutent et forment davantage de personnel «para légal » et semi-professionnel qui ne peut prétendre accéder au rang d'associé. Elles délèguent davantage d'autorité et de responsabilités à des non-avocats. Elles diversifient le champ de leurs activités, développant parfois des départements qui rendent à leurs clients des services qui n'ont plus rien de juridique (comptabilité, conseil financier, lobbying, etc.). Elles créent enfin des statuts intermédiaires entre stagiaires et associés, manipulant ainsi dans les faits la règle du up or out.

Bien que l'on dispose de peu d'informations très précises sur le partage des profits (ou compensation) entre associés copropriétaires de la firme, on connaît l'éventail des critères auxquels les firmes ont recours, de manière très variable, pour prendre ces décisions (Gilson et Mnookin, 1985). Les systèmes de redistribution varient entre deux extrêmes, la plupart des firmes se situant entre deux. Le premier système est basé sur l'ancienneté (lockstep seniority) : dès l'accession au rang d'associé, l'associé reçoit un pourcentage des profits qui croît au fil des années jusqu'à atteindre un certain plafond, d'habitude au bout d'une quinzaines d'années. Les firmes qui donnent la priorité à ce critère cherchent à se protéger des conflits internes en prenant en compte le «mérite », par exemple au moyen d'un système de boni. Le second système est basé presque exclusivement sur la productivité (nombre d'heures facturées, nombre de clients ramenés, responsabilités administratives ${ }^{5}$ ). D'ordinaire, une commission chargée du partage des profits (dont la composition est un enjeu de pouvoir important) alloue un pourcentage à chaque associé, ceci suivant une formule préétablie ou en fonction de critères plus complexes (faisant intervenir, par exemple, l'adéquation entre objectifs établis pour chacun par la planification stratégique et les résultats obtenus). Dans la mesure où la gestion et le partage du capital humain font aussi intervenir de la valeur difficilement mesurable, ce type de commission opère, d'une manière ou d'une autre, des ajustements à la hausse ou à la baisse. Dans certains cas, le reste des associés ne sait même pas avec précision comment la commission est parvenue à une distribution donnée. Il est difficile aussi de savoir, de l'extérieur, avec quelle fréquence des décisions de ce type sont contestées par des associés qui
5. A ma connaissance, les firmes qui fonctionnent exclusivement sur le principe de la rétribution d'un seul type de contribution (par exemple les systèmes du type eat what you kill) sont rares. 


\section{E. Lazega}

Collégialité et bureaucratie dans les firmes américaines d'avocats d'affaires s'estiment lésés. Entre ces deux grandes méthodes, la plupart des systèmes tiennent compte de plusieurs critères et pratiquent une pondération. Mais à mesure que les firmes adoptent une attitude entrepreneuriale, les systèmes de redistribution donnent davantage de poids à des critères comme la productivité et l'apport en clientèle qu'à l'ancienneté.

La plupart des grandes firmes américaines pratiquent depuis les années 1980 un marketing systématique qui n'a plus la discrétion et l'informalité auxquelles les contraignaient le code de déontologie et le fonctionnement même d'un marché basé sur le réseau (au sens de capital relationnel) et la confiance (Karpik, 1989). Pour des firmes qui cherchent à rester general practice dans un contexte qui encourage la croissance par la spécialisation, et où le client est souvent aussi représenté par d'autres firmes, toute une partie de la planification stratégique devient une forme d'ajustement au marché et de négociation permanente avec la clientèle. Traditionnellement, on considérait que le succès est basé sur la visibilité d'avocats individuels, leur insertion dans la communauté et la qualité de leur travail. Aujourd'hui, l'effort de marketing procède d'analyses plus systématiques de la demande, du repérage de clients particulièrement désirables, des décisions à moyen terme concernant le développement de départements dans les domaines du droit les plus lucratifs (avec les implications qu'ont ces décisions pour le recrutement de stagiaires), l'image de la firme, etc. Dans les firmes les plus commerciales, le marketing est organisé aux niveaux individuel, départemental (par domaine du droit) et global. Dans certaines firmes, les efforts de marketing sont comptabilisés au moment du partage des profits; par exemple, on rémunère les avocats qui ont réussi à se placer dans plusieurs conseils d'administration. Reste que, malgré cette infrastructure, il devient difficile pour des grandes firmes bien établies de se différencier vraiment les unes des autres au cours de ces campagnes de publicité.

Parmi les sujets les plus sensibles aujourd'hui dans les firmes américaines d'avocats d'affaires, on trouve la question de l'évaluation du travail de chaque associé par ses pairs (peer review). L'évaluation périodique des résultats et de la productivité de chaque associé au cours, par exemple, des réunions du partnership ou des sessions de la commission financière constituent des formes traditionnelles d'évaluation des contributions. Souvent, les firmes délèguent aux directeurs de département la responsabilité de surveiller informellement la qualité même du travail des associés et d'aider leurs collègues victimes de problèmes personnels qui interfèrent avec leur travail. Avec la croissance des firmes et sous la pression des assurances (malpractice insurance), il est fréquent aujourd'hui de rencontrer des commissions spéciales chargées de relire et d'approuver les opinions envoyées aux clients et engageant la firme toute entière, ou encore d'exiger des associés qu'ils suivent annuellement un nombre fixe d'heures de recyclage ou de formation continue. Mais certaines firmes introduisent de 
nouveaux mécanismes d'évaluation périodiques, par exemple une comparaison des performances des associés avec les objectifs qu'ils se sont fixés. Des débats s'instaurent dans certaines firmes sur le nombre d'heures que les associés devraient être obligés de facturer, c'est-à-dire sur ce qu'il faut considérer comme une contribution acceptable. Les sanctions prévoient par exemple le blocage de l'associé au palier où il se trouve dans le partage des bénéfices, ou d'autres arrangements temporaires. Dans les firmes où le critère de l'ancienneté est important au moment du partage des profits, ce moyen de contrôle est perçu comme un substitut à l'incitation à la productivité que peut constituer le couplage des contributions et des rétributions.

Les méthodes d'évaluation du travail des associés peuvent aller plus loin encore. Certaines firmes prévoient des systèmes de contrôle qui incluraient un échantillonnage des dossiers de l'associé, des entretiens avec des clients et du personnel de la firme. La mise en œuvre de ce type de contrôle n'est pas encore véritablement engagée, et son avenir n'est pas certain. Pour des raisons qui découlent de la nature collégiale de ce type d'organisation (Waters, 1989), certains associés perçoivent le contrôle de la qualité de leur travail comme une atteinte à leur indépendance et une contestation de la qualité de leur jugement. Les objections soulevées par de nombreux associés contre ce type de contrôle sont nombreuses. Pour beaucoup, une évaluation de ce type ne devrait avoir lieu que lorsqu'un client proteste contre la qualité insuffisante du travail; les problèmes viendraient plutôt des associés qui ne travaillent pas assez, ou qui acceptent de se charger d'affaires indésirables. Pour d'autres, l'évaluation et le contrôle devraient rester informels et incorporés dans les procédures d'ouverture des dossiers et de constitution des équipes. De plus, un système formel d'évaluation des pairs serait trop coûteux (en temps) et difficile à mettre sur pied (certaines spécialités n'étant représentées dans la firme que par un seul associé). Les instances de gestion cherchent donc à désamorcer les aspects menaçants de ces évaluations et à les dédramatiser, par exemple en les faisant passer pour des manières de repérer les associés en difficulté.

Les changements organisationnels ont aussi un effet sur le recrutement, la formation, le travail et l'évaluation des stagiaires, ainsi que sur leurs chances d'accéder au rang d'associé. Pour utiliser leur personnel efficacement et pour répondre à la demande du marché, les firmes développent une division du travail très complexe et idiosyncratique. Mais pour attirer les meilleurs avocats débutants et maintenir leur enthousiasme au travail, elles cherchent à conserver une aura de liberté et de choix individuels. Les carrières des avocats sont ainsi pré-orientées de différentes manières indirectes pour répondre aux besoins de la firme. Bien que cette pré-orientation ait toujours existé, les firmes d'aujourd'hui gèrent ces pressions de manière plus indirecte, en les définissant pour les stagiaires davantage comme des opportunités (Nelson, 1988). En choisissant très tôt des spécialités et 


\section{E. Lazega}

Collégialité et bureaucratie dans les firmes américaines d'avocats d'affaires

6. La formation des stagiaires, souvent jugée insuffisante par les associés et les stagiaires euxmêmes, se fait sur le tas; elle dépend donc beaucoup des capacités pédagogiques des associés, mais aussi des relations et de la communication -pas toujours directe et ouverte du fait des enjeux de carrière- entre stagiaires et associés.

7. Un non-equity partner, par exemple, est un associé qui ne partage pas les bénéfices de la firme et ne vote pas pour les grandes décisions prises par l'assemblée des associés. sous-spécialités, les stagiaires jouent leurs chances de devenir associés sur le taux de croissance du domaine qu'ils ont choisi. La plupart des firmes structurent les carrières des stagiaires au moyen de commissions (souvent deux, l'une côté litigation, l'autre côté corporate), de directeurs de départements, de «parrainage » plus ou moins réussis par des associés. Ces commissions surveillent la distribution du travail aux stagiaires, établissent les priorités et évaluent leur productivité, bien que dans les grandes firmes polycentriques ce contrôle leur échappe très vite, et que l'évaluation des stagiaires par ces commissions ne devienne problématique. Les directeurs de département jouent dès lors un rôle très important pour les stagiaires : ce sont eux en général qui, de fait, distribuent le travail, couplent associés et stagiaires, organisent l'évaluation bi-annuelle et la défense du dossier d'un stagiaire devant l'assemblée des associés. La qualité du travail même des stagiaires est généralement surveillée par l'associé responsable du dossier ou par un stagiaire plus ancien travaillant sur la même affaire.

Les firmes cherchent à recruter dans les facultés de droit les plus réputées et consacrent beaucoup de ressources à leurs stagiaires (en salaires élevés si ce n'est en formation suivie ${ }^{6}$ ), ce qui souligne l'importance du rôle économique qu'ils jouent. Sans les compensations que représentent ces salaires élevés, la perspective d'accéder au rang d'associé et parfois le prestige d'appartenir à une grande firme réputée régionalement ou nationalement, les stagiaires n'accepteraient pas la règle du jeu du up or out. Reste que les carrières qui suivent le tracé établi par le système Cravath sont de plus en plus réservées à un nombre limité de stagiaires. Le principe selon lequel il n'y a pas de recrutement sans promotion n'a plus rien d'absolu. Aujourd'hui, la plupart des cabinets ont peu de possibilités de diversification et font face à des contraintes importantes en matière d'expansion. En période de crise, même lorsque le volume de travail augmente dans certaines spécialités (contentieux, environnement, faillites), les cabinets licencient des stagiaires, ou en tout cas n'en recrutent plus au même rythme qu'au cours des années 1980 (Galanter et Palay, 1991). Malgré cela, l'organisation de la firme autour de la promotion au rang d'associé (comme mécanisme de protection du capital humain et social, de contrôle et de motivation des stagiaires) ne disparait pas en tant que modèle. La plupart des firmes créent de nouvelles positions, pour les associés comme pour les stagiaires, qui peuvent être à la fois permanentes et salariées ${ }^{7}$. En conséquence, elles s'attendent à ce que ce changement affecte le comportement des stagiaires et le rende plus ouvertement « opportuniste ». Les chances de devenir associé s'affaiblissant encore davantage que par le passé, il en résulte une grande incertitude quant à l'avenir de ce système. 
Dans ces organisations professionnelles souvent caractérisées par leur élitisme et, auparavant, par leur informalité, des résistances se manifestent contre les changements en cours. Ces résistances perdurent d'autant plus que la bureaucratisation, on l'a vu, est limitée par plusieurs facteurs. Nelson (1988) en retient trois : l'existence de normes professionnelles, la nature du travail et des tâches à accomplir, les exigences formulées par la clientèle.

D'abord, les normes du professionnalisme (par exemple l'appel aux valeurs d'indépendance, d'imagination, de service public) fonctionnent toujours comme une culture de motivation au sein même de structures très formalisées. Ensuite, pour faire concurrence aux avocats des grandes entreprises (in-house lawyers), les firmes doivent pouvoir prendre en charge des dossiers volumineux et complexes. Pour ce faire, elles développent, comme on l'a indiqué plus haut, de nombreuses spécialités et des capacités de mobilisation et de coordination d'équipes improvisées. Mais elles doivent en même temps rester d'une certaine manière artisanales puisque le travail à accomplir n'est pas un travail de routine, engage de grosses sommes et des enjeux stratégiques importants, exige de l'innovation en matière de technique juridique et du tact pour gérer des situations politiquement délicates au sein même des entreprises qui retiennent les services de la firme. Pour accomplir ces tâches, les cabinets doivent compter sur le jugement, le talent et la discrétion de leurs experts, et donc s'effacer, en apparence tout au moins, devant les normes de la profession qui s'appliquent à l'organisation du travail. Elles orchestrent donc un ensemble d'activités très spécialisées avec un minimum de règles et de procédures. Celles-ci tentent de contrôler le contexte organisationnel du travail, non pas directement le contenu du travail lui-même (Freidson, 1986). Enfin, la relation avec le client reste imprévisible dans la mesure où les entreprises utilisent souvent plusieurs firmes à la fois et imposent des exigences qui constituent souvent une source d'instabilité pour des procédures bien établies. La rationalisation des firmes d'avocats est limitée par les tensions qui apparaissent ainsi entre autorité managériale et responsabilité vis-à-vis du client. Bien plus, l'autorité nécessaire à la formulation des politiques de la firme reste fragile et dépend de la capacité des associés à attirer de gros clients, à créer un consensus parmi les associés sur la manière de conduire les affaires de la firme, et à entretenir la motivation des associés et stagiaires en matière de qualité du travail. C'est ce que Nelson (1988) appelle la nature «faible » de l'autorité dans les firmes d'avocats, qui n'est pas sans ressemblance avec celle que l'on trouve dans d'autres organisations collégiales.

Les résistances contre la bureaucratisation reposent aussi sur des mécanismes qui relèvent d'un niveau plus fondamental - d'une logique sociale de l'action professionnelle - et qui, paradoxalement, 


\section{E. Lazega}

Collégialité et bureaucratie dans les firmes américaines d'avocats d'affaires

8. La question de l'intégration est omniprésente dans les entretiens avec les responsables de ces firmes, en particulier dans leurs propos sur

l'« opportunisme ». Gilson et Mnookin (1985) identifient trois formes de comportement considéré comme " opportuniste » (parce que menaçant la manière dont se partagent les profits et le capital humain de la firme) à la base des tendances centrifuges à l'œuvre dans ce type d'organisation professionnelle: la baisse de productivité (shirking), la lutte pour s'approprier des ressources ou des clients (grabbing) et le changement d'organisation (leaving). Face à l'opportunisme, les associés considèrent la collégialité comme un code de conduite relationnelle et informelle qui devrait promouvoir la confiance et la coopération, assurer l'intégration de la firme et faciliter la transition en période de changements organisationnels et de crise économique. constituent une condition d'existence d'organisations de ce type. Les mécanismes d'intégration formels et informels sont à cet égard instructifs ${ }^{8}$. La structure formelle de ces organisations, basée sur le système de prises de décisions par commissions successives, a une capacité intégratrice à condition qu'elle s'accompagne de normes qui font peser sur ces commissions une forte pression vers le consensus (Musselin, 1990). La polyvalence des relations collégiales, les réseaux de collaboration, d'« amitié » et d'échanges de conseils à l'intérieur de la firme ont, eux aussi, une capacité intégratrice : en effet, ils permettent de modérer ou de mieux gérer d'éventuels conflits entre membres, dus notamment à la distance géographique entre bureaux (qui correspond à une différence entre marchés aux exigences différentes), aux écarts de statut (stratification interne entre associés et stagiaires) et aux différences de spécialité (division du travail entre le contentieux et le conseil d'entreprise). De même, on a observé que les relations de collaboration peuvent se structurer d'une manière qui cherche à affaiblir la concentration du pouvoir dans la firme, par exemple un contrôle simultané de la clientèle et de la main d'œuvre. Un tel mécanisme crée une interdépendance entre associés et tend à empêcher l'éclatement de la firme en plusieurs firmes différentes (Lazega, 1992a, 1992b).

La résistance du modèle collégial s'appuie donc à la fois sur les limites de la rationalisation bureaucratique et sur les modalités d'auto-régulation sociale que l'on observe dans ce type d'association collégiale. Avant de décrire les tensions qui se développent entre cet équilibre organisationnel et certaines exigences de l'« éthique professionnelle », il est utile de mentionner l'émergence de nouvelles formes organisationnelles, moins bien connues que la précédente mais très représentatives, elles aussi, des changements que subit la pratique du droit et de son commerce aux États-Unis.

\section{De nouvelles formes organisationnelles}

Les méga-firmes internationales: On ne dispose d'aucune étude des méga-firmes internationales qui analyse leur organisation comme on le ferait, par exemple, d'une multinationale industrielle. Les travaux exploratoires comme ceux d'Ackermann et Parthenay (1992; voir aussi à l'intérieur de ce numéro) montrent les difficultés rencontrées par une firme américaine implantée à Paris qui mélange des avocats américains et français. La synthèse de bureaucratie et de collégialité qui caractérise le fonctionnement formel et informel de ces mégafirmes intercontinentales est mal connue. La bureaucratisation y estelle plus poussée que dans les firmes de taille moyenne? L'étude des relations entre agences ou filiales d'une même firme reste à faire : quelles nationalités pour les postes-clés, quelle autonomie pour les bureaux ou filiales, quels systèmes de prise de décision et de rémunération, quels critères d'équité dans la gestion des ressources humai- 
nes et des carrières, quels normes professionnelles pour gérer la concurrence et la coopération avec d'autres firmes, quelles stratégies de protection de leur capital social et d'expertise, quelles formes de division du travail? Il en va de même pour la comparaison entre firmes internationales et réseaux de firmes nationales (ou autres formes flexibles d'association). Dans la mesure où leurs formes organisationnelles résultent de la présence de forces parfois opposées de différents marchés, de différents États et de différentes professions juridiques, une telle étude serait d'une grande importance pour le débat sur l'internationalisation du droit (Dezalay, 1992).

Les franchiseurs: Si la spécificité des méga-firmes anglosaxonnes et internationales a été très peu prise en compte par la littérature, le fonctionnement des firmes basées sur le principe de la franchise a été remarquablement documenté par Seron (1992). La différence entre ces firmes et la general practice firm classique est d'abord liée à la nature des services juridiques fournis. Les franchiseurs servent des clients individuels, éventuellement des petites et moyennes entreprises, mais pas de grandes sociétés.

Depuis que les firmes américaines ont obtenu le droit de faire connaître au public le prix de leurs services (1977), certaines entreprises utilisent systématiquement la publicité au niveau national, mais fonctionnent au niveau local suivant le principe des franchises (comme Jacoby and Meyers ou Hyatt Legal Services) ou des assurances (comme Nationwide Prepaid Plan ou Signature Group). Cette forme d'organisation permet aux associés de puiser dans le marché des petites et moyennes entreprises. Elle combine une bureaucratisation poussée (standardisation et routinisation des tâches venant d'« en haut ») avec une relation au client de type traditionnelle et personnalisée (Seron, 1992).

Ces nouvelles structures offrent aux avocats une gestion de la partie administrative et publicitaire de leur pratique. Elles sont plus bureaucratisées que les firmes du type Cravath car leur gestion, qui assure une part importante de la productivité de l'entreprise, est contraignante. En échange, les associés peuvent davantage fonctionner comme des avocats « à l'ancienne », sur le modèle du notable local qui jouit d'une autonomie accrue vis à vis de ses clients. Seron (1992) voit dans l'émergence de ces nouvelles formes de pratique une redivision du travail juridique entre entrepreneurs, managers et professionnels. Ces formes caractérisent des «orientations » différentes dans la profession juridique américaine, qui ajouteraient encore à sa segmentation et à son «malaise », tout en reposant la question de sa cohésion.

Les partnerships multidisciplinaires : Les partnerships multidisciplinaires cherchent à devenir des one-stop shop et à offrir à une clientèle d'entreprises plusieurs services de conseil (juridique, mais aussi financier, de marketing, de comptabilité, de lobbying, etc.). Sous des formes variées, les firmes multidisciplinaires existent en Europe 


\section{E. Lazega}

Collégialité et bureaucratie dans les firmes américaines d'avocats d'affaires
9. Voir par exemple, au sujet de cette question, Abel (1989a et 1989b), Pue (1989), Kritzer (1991). et aux États-Unis. La cohabitation de professionnels différents au sein d'une même firme, les relations entre la culture organisationnelle et les cultures professionnelles représentées, les compromis éthiques émergeant des conflits d'intérêts, sont autant de sujets très peu étudiés par la littérature (Flood, 1993 à paraitre). Pourtant certaines formes de multidisciplinarité remettent en question les statuts et protections actuelles des professions. Le problème est essentiellement celui de la régulation de ces sociétés, dont les membres ne sont pas nécessairement surveillés par une seule instance de contrôle. Les organisations professionnelles américaines et européennes (comme par exemple celle des juristes et celle des comptables) ne parviennent pas à forger de consensus sur la régulation de leurs pratiques et sur les règles de la concurrence qu'elles devraient respecter (Abbott, 1988). Il est possible que, tout comme pour les sociétés multinationales, les associations ou réseaux de firmes de professions différentes soient à la longue des solutions plus lucratives et plus viables que le fait de devenir soi-même une firme multidisciplinaire.

\section{Bureaucratie, collégialité et professionnalisme}

La section consacrée à la résistance du modèle collégial donne quelques exemples de la manière dont la profession juridique, au travers de ses normes, contribue à structurer les firmes d'avocats d'affaires. Mais à l'inverse, certains aspects du fonctionnement interne de ces firmes décrivent aussi un effet en retour de ces organisations sur la profession et ses normes. Des auteurs comme Galanter et Palay (1991) ou Nelson, Trubek et Solomon (1992), après bien d'autres ${ }^{9}$, déplorent un "déclin du professionnalisme » américain ou du moins une « redéfinition » des normes de la profession. Il est possible d'affirmer que la profession juridique, en tant qu'acteur collectif, a peu de contrôle direct sur les conditions dans lesquelles s'exerce le commerce du droit des affaires. Mais il est tout aussi important d'évaluer l'influence des grandes firmes sur la profession, notamment à travers ce processus de redéfinition des règles du professionnalisme.

$\mathrm{Du}$ fait de difficultés de méthode et d'accès, peu de recherches empiriques ont été consacrées à ce processus. Le travail de Nelson (1988) suggère que les associés qui sont en position de pouvoir dans ces grandes firmes sont aussi en position d'imposer leur conception du professionnalisme. Un autre exemple de redéfinition des règles de l'éthique réside dans l'attitude des firmes vis à vis du problème de la concurrence entre elles et des conflits d'intérêts - auquel elles se trouvent de plus en plus confrontées. En effet, en contrepartie des privilèges qui lui sont accordés par l'État, la profession est censée surveiller le fonctionnement du marché du conseil et de la représentation juridique, marché dont ses membres sont eux-mêmes des parties prenantes. Pour promouvoir une gestion éthique de la concurrence entre avocats, les firmes gèrent les conflits d'intérêts au moyen de 
deux procédés : la décision d'auto-disqualification et l'implantation de «murs chinois » 10 . Une étude empirique du processus de la décision d'auto-disqualification et de l'efficacité des murs chinois n'a jamais été tentée. Cependant, le fonctionnement interne des firmes suggère que ces procédés sont peu crédibles. L'efficacité de ces «murs » en tant que dispositif organisationnel est douteuse du fait de l'appartenance des membres à des réseaux (internes à la firme) d'échanges d'informations et de conseils dont ils ne peuvent se détacher de manière systématique. L'auto-disqualification est souvent peu vraisemblable parce que le régime de contrôle informel de ces firmes n'est pas en mesure de forcer un associé à s'auto-disqualifier en faveur d'un autre sans créer de conflits majeurs qui remettent en question l'intégration de l'organisation (Lazega, 1992a, 1993). En effet, dans ce type d'organisation collégiale, les " contrôleurs » sont eux-mêmes fortement dépendants des "contrôlés », et bien conscients du sort réservé aux « redresseurs de tort»11. A défaut donc d'une solution éthique au problème de la représentation multiple, les grandes firmes américaines cherchent à faire redéfinir par le barreau américain la notion même de conflit d'intérêts, et ceci d'une manière qui permet d'ignorer une grande partie de ces conflits sans risquer la disqualification par le juge. Les débats sur ces questions sont en cours à l'American Bar Association.

Que les règles du professionnalisme se négocient ainsi dans les organisations où se pratique le droit des affaires fait apparaître, nous semble-t-il, une limite importante de l'auto-régulation ${ }^{12}$ par la profession et une incertitude quant au fonctionnement futur du marché des services juridiques (Karpik, 1989). Mais ce paradoxe de la logique d'action professionnelle relève aussi, plus généralement, d'une limite actuelle du contrôle social sur la vie organisationnelle (Reiss, 1988).

\section{Des formes organisationnelles qui se diffusent en Europe?}

La structure traditionnelle des firmes américaines d'avocats d'affaires s'est graduellement modifiée sous la pression des processus internes de croissance, de la logique d'action professionnelle et du marché. Du point de vue des différences de structure formelle (importance de la départementalisation, arrangements polycentriques) et de politique de gestion (concernant la distribution des dossiers, le partage des bénéfices entre associés, le marketing, l'évaluation de la qualité du travail, la gestion des stagiaires), les firmes d'aujourd'hui représentent chacune une variante plus ou moins éloignée du modèle initial (le système Cravath). Malgré ces changements et cette diversité formelle, toutes font face aux mêmes problèmes : perte du contrôle de leur croissance, nouvelle redéfinition du professionnalisme, déve-
Droit et Société 23/24-1993

10. Il s'agit d'un système de sécurité concernant les flux d'in-

formations. Son aspect essentiel est une régulation de la communication intra-

organisationnelle qui isole les employés ou les membres les uns des autres lorsqu'ils travaillent sur des cas qui créent un conflit dû à la représentation multiple. Ces membres ne sont pas supposés communiquer. Cette règle est parfois appelée un « Chinese wall » ou " mur chinois ». Les études d'avocats utilisent de telles compartementalisations pour garantir le fait que la partie adverse n'a pas accès à des informations confidentielles (Hamermesh, 1986; Morgan, 1987), donc pour éviter les conflits d'intérêts dits

« politiques » ou

« commerciaux ». Ce mécanisme d'« auto-régulation » s'appuie sur l'éthique pour protéger la confiance du client, et constitue un moyen reconnu -mais contesté- d'éviter la disqualification par le juge (voir encore à ce sujet Hazard, 1987, 1988).

11. La collégialité a toujours été considérée comme une garantie de professionnalisme, d'objectivité ou de justice dans la prise de décisions importantes comme le recrutement ou le partage de ressources: elle permet, par exemple, de diminuer les risques que ces dernières ne se prennent " à la tête du client ». Une approche empirique de la question suggère que le régime de contrôle entre associés n'est pas toujours capable d'assurer le type de contrôle social que Weber attendait du système collégial, en particulier dans le domaine éthique. Ceci suggère que l'application des règles éthiques dans un contexte d'autorégulation est aussi complexe que dans des contextes où la régulation est mise en œuvre par des agents externes (Hawkins, 1984; Lascoumes, 1990). 12. Voir sur ce sujet, par exemple, Gillers et Dorsen (1985), Powell (1985), Dingwall et Fenn (1987), Smith (1989), Eidenmuller (1990), Braithwaite et Ayres (1991). 


\section{E. Lazega}

Collégialité et bureaucratie dans les firmes américaines d'avocats d'affaires

13. Les grandes firmes londoniennes de sollicitors sont, de longue date, construites sur ce modèle.

14. Si les États et barreaux nationaux les y autorisent, certaines firmes pourraient même abandonner la forme du partnership -essentiellement pour échapper aux contraintes de la responsabilité collective (liability)- pour celle de société à responsabilité limitée. Ces firmes modifieraient ainsi, en partie, les rapports entre organisation, avocats et clientèle, mais aussi les formes de régulation auxquelles elles seront soumises.

15. Voir par exemple Karpik (1985, 1989) et Dezalay (1992) pour le cas français; Blankenburg et Schultz (1988), Günther (1991) et Rogowski (1993) pour le cas allemand. loppement d'une culture «entrepreneuriale », nouvelles formes de relations collégiales.

Le modèle Cravath, dont l'avenir est incertain aux États-Unis, se diffuse aujourd'hui dans les cabinets d'avocats d'affaires en Europe continentale 13. L'implantation des firmes anglo-saxonnes sur le continent et la croissance des firmes d'origine continentale constituent un phénomène général en Europe. L'ouverture et l'internationalisation (intra-européenne) des marchés et le processus de construction politique de l'Europe transforment les professions traditionnelles du droit et les politiques de régulation de la profession. Une nouvelle profession juridique européenne se met actuellement en place (Karpik, 1985; Dezalay, 1992). Elle tend vers davantage de spécialisation, mais aussi vers davantage d'homogénéisation des professions nationales et de leurs codes d'éthique (Skordaki et Sutton, 1991). Malgré les difficultés de fonctionnement du marché des services juridiques et de la régulation de la concurrence entre firmes, malgré l'ambiguïté des réponses des professions juridiques au caractère de plus en plus compétitif de ce marché, les firmes de taille moyenne se développent en Europe parce qu'elles jouent un rôle spécifique dans la restructuration économique actuelle (Dezalay, 1992; Whelan et McBarnet, 1992). On ne sait pas encore si ces transformations seront plus ou moins semblables à une forme d'« américanisation » (Siegrist, 1992). Les variantes européennes ${ }^{14}$ du modèle Cravath sont encore peu décrites (Ackermann et Parthenay, 1992; Lee, 1992; Whelan et McBarnet, 1992) et la légitimité même de cette comparaison fait aujourd'hui l'objet de spéculations. 15

Quel que soit l'avenir de ce modèle, les questions que les sociologues se posent au sujet des firmes américaines valent aussi pour les firmes européennes. Les firmes continentales prestataires de conseil juridique aux grandes entreprises s'associent parfois avec des études américaines géantes qui veulent s'étendre. Du coup, elles doivent se réorganiser, se soumettre par exemple à des tests (screening) relatifs aux conflits d'intérêts, tout en essayant de rester "à l'échelle humaine », de garder l'apparence rassurante de la tradition. D'autres firmes européennes intra- et inter-nationales fusionnent, s'associent ou se regroupent de manière plus ou moins contraignante en « réseaux » pour faire face à la concurrence américaine, notamment à Bruxelles. Pour ces firmes, la question de savoir comment se structurer d'une manière qui évite les conflits d'intérêts, qui soit en même temps crédible pour la clientèle et efficace pour le contrôle de la compétition entre firmes est une question d'actualité.

Dans ces conditions, il est difficile de prédire le type de firme qui deviendra dominant en Europe. La connaissance des formes organisationnelles que prend la pratique du droit des affaires dans le monde anglo-saxon permet de mettre en perspective et, peut-être, d'anticiper des changements en cours sur le continent européen. Cependant, des formes organisationnelles nouvelles pourraient apparaître avec l'émer- 
gence d'une profession juridique européenne faite de professions juridiques nationales, à l'histoire et aux pratiques très différentes, qui s'organisent pour se faire représenter auprès de la Commission européenne chargée de leur régulation commune. Les formes organisationnelles adoptées par ces firmes résument en elles-mêmes l'équilibre qui résulte de la coexistence des forces parfois opposées du marché, de l'État et de la profession. Les formes de demain dépendront donc aussi des compromis auxquels seront parvenus les professions juridiques, les marchés et les États européens.

\section{Bibliographie}

Abel R. et Lewis Ph. (eds) 1988, 1989, Lawyers in Society, volume I : The Common Law Countries, volume II : The Civil Law Countries, volume III : Comparative Theories, Berkeley, University of California Press.

Abel R.

1989, « The Legal Profession in Turmoil ", Modern Law Review, vol. 52.

1989, American Lawyers, New York, Oxford University Press.

\section{Abbott A.}

1988, The System of Professions : An Essay on the Division of Professional Labor, Chicago, Chicago University Press.

\section{Ackermann W. et Parthenay S.} 1992, « The integration of French lawyers into the Paris office of a large American law firm », Communication présentée à la Conférence européenne sur les professions juridiques, Aix-en-Provence.

\section{Blankenburg E. et Schultz U.}

1988, « German advocates : a highly regulated profession ", in R.Abel and Ph.Lewis (eds), Lawyers in Society, vol.2 : The
Civil Law World, Berkeley, University of California Press.

\section{Bourricaud F.}

1961, Esquisse d'une théorie de l'autorité, Paris, Plon.

\section{Braithwaite J. et Ayres I.}

1991, « Convergence in models of regulatory strategy : from deregulation to responsive regulation », Communication présentée au congrès de la Law and Society Association, Amsterdam.

\section{Davies C.}

1983, «Professionals in bureaucracies : the conflict thesis revisited ", in R. Dingwall et P. Lewis (eds), The Sociology of the professions, London, Macmillan.

\section{Dezalay Y.}

1992, Marchands de droit : La restructuration de l'ordre juridique international par les multinationales du droit, Paris, Fayard.

\section{Dingwall R. et Fenn P.}

1987, « A respectable profession? Sociological and economic perspectives on the regulation of professional services », International Review of Law and Economics, vol. 7. 


\section{E. Lazega}

Collégialité et bureaucratie dans les firmes américaines d'avocats d'affaires

\section{Eidenmuller $\mathbf{H}$.}

1990, « Deregulating the Market for Legal Services in the European Community », Modern Law Review, vol. 53.

\section{Flood J.}

1993, « The Legal and Business Professions of Europe in 1992 and Multidisciplinary Practice », in Y.Dezalay et D.Sugarman (eds), Professional Competition and the Social Construction of Markets, London, Routledge.

\section{Freidson E.}

1986, Professional Powers, Chicago, University of Chicago Press.

Galanter M. et Palay T. 1991, Tournament of Lawyers : The Transformation of the Big Law Firm, Chicago, University of Chicago Press.

\section{Gillers S. et Dorsen N.}

1985, Regulation for Lawyers : Problems of Law and Ethics, Boston, Harvard University Press.

Gilson R.J. et Mnookin R.H. 1985, « Sharing Among Human Capitalists : An Economic Inquiry into the Corporate Law Firm and How Partners Split Profits », Stanford Law Review, 37 : 313-392.

1989, " Coming of Age in the Corporate Law Firm : The Economics of Associate Career Patterns ", Stanford Law Review, 41 : 567-595.

Groot-Van Leeuwen L.E. de 1992, "Lawyers' Ethics in Transition », Communication présentée à la Conférence européenne sur les professions juridiques, Aix-en-Provence.

\section{Guenther K.}

1991, « The German Transformation and Traditional Practice », Communication présentée à la conférence : « the New European Legal Profession and the American Legal Challenge », Amsterdam, juin 1991.

Halliday T.

1987, Beyond Monopoly : Lawyers, State Crisis, and Professional Empowerment, Chicago, Chicago University Press.

Hamermesh F. W. 1986, «In Defence of a Double Standard in the Rules of Ethics : A Critical Reevaluation of the Chinese Wall and Vicarious Disqualification », Journal of Law Reform, 20 : 245-277.

\section{Hawkins $\mathrm{K}$.}

1984, Environment and Enforcement, Oxford, Oxford University Press.

Hazard G. C. Jr.

1980, Ethics in the Practice of Law, New Haven, Yale University Press.

1987, « When there are Conflicts over Conflicts », The National Law Journal, vol. 10, 19 oct, p. 13.

Hazard G.C.Jr. et Rhode D.L. (eds) 1988, The Legal Profession: Responsibility and Regulation, New York, The Foundation Press.

Heinz J.P. et Laumann E.O. 1982, Chicago Lawyers : The Social Structure of the Bar, New York, Russell Sage Foundation and American Bar Foundation. 
Kagan R.A. et Rosen R.E.

1985, « On the Social Significance of Large Law Firm Practice ", Stanford Law Review, 37 : 399-443.

\section{Karpik L.}

1985, « Avocat : une nouvelle profession? ", Revue Française de Sociologie, 26 : 571600.

1989, «L'Économie de la qualité », Revue Française de Sociologie, 30 : 187-210.

\section{Kritzer H.M.}

1991, " Abel and the Professional Project : The Institutional Analysis of the Legal Profession ", Law and Social Inquiry, $16: 529-552$.

\section{Lascoumes P.}

1990, « Normes juridiques et mise en œuvre des politiques publiques ", L'année sociologique, $40: 43-71$.

\section{Laumann E.O. et Heinz J.P.} 1977, « Specialization and Prestige in the Legal Profession : The Structure of Deference ", American Bar Foundation Research Journal, 1 : 155-216.

\section{Lazega E.}

1992a, « Analyse de réseaux d'une organisation collégiale : les avocats d'affaires », Revue française de sociologie, 33 : 559-589.

1992b, Bureaucratie, collégialité et professionnalisme. Étude de la structure formelle et informelle d'une firme américaine d'avocats d'affaires, Rapport de recherche, Genève, Fonds National Suisse de la Recherche Scientifique.
1993 (à paraître),

« Concurrence et auto-

régulation professionnelle : une approche organisationnelle des conflits d'intérêts dans les firmes américaines d'avocats d'affaires ».

\section{Lazega E. et Vari S.}

1992, « Acteurs, cibles et leviers : analyse factorielle de réseaux de contrôle dans une firme américaine d'avocats d'affaires », Bulletin de Méthodologie Sociologique, 37 : 4151.

Lee R.G.

1992, «From Profession to Business : The Rise and Rise of the City Law Firm », Journal of Law and Society, Special issue on Tomorrow's Lawyers, edited by Philip A.Thomas, 19 : 31-48.

\section{Mann K.}

1985, Defending White-Collar Crime : A Portrait of Attorneys at Work, New Haven, Yale University Press.

\section{McCahery J. et Picciotto S.}

1992, "Creative Lawyering and the dynamics of the organization of the legal profession », Communication présentée à la conférence « Juristes et Comptables en Europe », Paris, 20 novembre 1992.

\section{Morgan T.D.}

1987, "Screening the Disqua-

lified Lawyer : The Wrong

Solution to the Wrong Problem », Altheimer Lecture at the University of Arkansas at Little Rock School of Law. 


\section{E. Lazega}

Collégialité et bureaucratie dans les firmes américaines d'avocats d'affaires

\section{Musselin C.}

1990, «Structures formelles et capacités d'intégration dans les universités françaises et allemandes ", Revue française de sociologie, 31 : 439-461.

\section{Nelson R.L.}

1988, Partners With Power: The Social Transformation of the Large Law Firm, Berkeley, University of California Press.

\section{Nelson R.L., Trubek D.M. et So-} lomon R.L. (eds.)

1992, Lawyers' Ideals / Lawyers' Practices : Transformations in the American Legal Profession, Ithaca, NY, Cornell University Press.

\section{Osiel M.J.}

1990, « Lawyers as Monopolists, Aristocrats, and Entrepreneurs », Harvard Law Review, vol. 103.

\section{Powell M.J.}

1985, « Developments in the Regulation of Lawyers », Social Forces, 64 : 281-305.

1991, « The Changing Form of Partnership in Large Law Firms », Communication présentée au congrès de la Law \& Society Association, Amsterdam.

Pue W.W.

1989, « Trajectories of Professionalism? Legal Professionalism after Abel », Manitoba Law Annual, p.57-92.

Reiss A. J. Jr.

1988, « The Control of Organizational Life », in Conflict and Integration : Comparative Law in the World Today, The Institute of Comparative Law in Japan, Chuo University.

\section{Rogowski R.}

1993, « German Corporate Lawyers », in Y.Dezalay et D.Sugarman (eds), Professional Competition and the Social Construction of Markets, London, Routledge.

\section{Scott W.R.}

1965, «Reactions To Supervision in a Heteronomous Professional Organization », Administrative Science Quarterly, $65: 65-81$.

\section{Seron C.}

1992, « Managing Entrepreneurial Legal Services : The Transformation of Small-Firm Practice », in R.L.Nelson, D.M.Trubek et R.L.Solomon (eds.), Lawyers' Ideals / Lawyers' Practices : Transformations in the American Legal Profession, Ithaca, NY, Cornell University Press.

\section{Siegrist $\mathrm{H}$.}

1990, «Die Genfer Advokaten im 19. und frühen 20. Jahrhundert », in S.Brändli et al. (eds), Schweitz im Wandel, Basel/Frankfurt am Main, Helbing \& Lichtenhahn, p. 229257.

1992, « Les Professionnels du droit continentaux : des modèles différents », Communication présentée à la conférence " Juristes et Comptables en Europe », Paris, novembre 1992.

\section{Skordaki E. et Sutton L.} 1991, European lawyers and the single market : A study carried out by the Conseil supérieur du notariat français, London, Law Society Research and Policy Planning Unit. 


\section{Smigel E.}

1969, The Wall Street Lawyer: Professional Organizational Man? $2^{\circ}$ ed, Bloomington, Indiana University Press.

\section{Smith R.}

1989, « The Green Papers and Legal Services ", Modern Law Review, vol. 52.

\section{Sterett S.}

1990, « Comparing Legal professions ", Law and Social Inquiry, $15: 363-384$.

\section{Stewart P.}

1975, «Professional Ethics for the Business Lawyer : The Morals of the Marketplace », $\mathrm{Bu}$ siness Lawyer, 31 : 463-468.

\section{Waters $\mathrm{M}$.}

1989, « Collegiality, Bureaucratization, and Professionalization : A Weberian Analysis ", American Journal of Sociology, $94:$ : 945-72.

\section{Weber $\mathrm{M}$.}

1978, Economy and Society, Berkeley, University of California Press.

\section{Whelan, C. et McBarnet, D.}

1992, "Lawyers in the Market : Delivering Legal Services in Europe », Journal of Law and Society, Special issue on Tomorrow's Lawyers, edited by Philip A.Thomas, 19 : 4968. 